\title{
Catheter ablation can prevent VT
}

Catheter ablation guided by electroanatomic mapping is now a viable treatment option to reduce episodes of recurrent ventricular tachycardia (VT) in patients who have experienced myocardial infarction. A multicenter study by William Stevenson et al. has shown that patients can be free of VT 6 months after ablation.

VT episodes can be terminated, but not prevented, using an implantable cardioverter-defibrillator. In patients with these devices, antiarrhythmic drug therapy is often administered to prevent VT; however, these agents have adverse effects and relatively poor efficacy.

Stevenson and colleagues evaluated 231 patients with recurrent episodes of monomorphic VT (median 11 in the preceding 6 months) caused by previous myocardial infarction. Ablation was carried out in all patients by means of a saline-irrigated catheter combined with electroanatomic mapping system to assist substrate mapping during sinus rhythm.
At the 6-month follow-up, 53\% of patients were free of VT, and the 6-month frequency of VT episodes was reduced from a median of 11.5 to 0 in the 142 patients who had an implantable cardioverter-defibrillator before ablation. A total of 7 deaths were recorded within 7 days of the procedure and the 1-year mortality rate was $18 \%$.

"The dramatic reduction of VT episodes after catheter ablation would likely be associated with an improved quality of life in the high-risk patients assessed in this study," says Hugh Calkins from the Johns Hopkins Hospital in the USA.

Lisa Richards

Original article Stevenson, W. G. et al. Irrigated radiofrequency catheter ablation guided by electroanatomic mapping for recurrent ventricular tachycardia after myocardial infarction: the multicenter thermocool ventricular tachycardia ablation trial. Circulation 118 , 2773-2782 (2009). 